\title{
Stochastic constrained control: trading performance for state constraint feasibility
}

\author{
Luca Deori, Simone Garatti, and Maria Prandini
}

\begin{abstract}
In this paper, we address finite-horizon control for a stochastic linear system subject to constraints on the control and state variables. A control design methodology is proposed where the appropriate trade-off between the minimization of the control cost (performance) and the satisfaction of the state constraints (safety) can be decided by introducing appropriate chance-constrained problems depending on some parameter to be tuned. From an algorithmic viewpoint, a computationally tractable randomized approach to find approximate solutions which are guaranteed to be feasible for the original chanceconstrained problem is proposed. A numerical example concludes the paper.
\end{abstract}

\section{INTRODUCTION}

Consider a linear system whose state $x_{t} \in \mathbb{R}^{n}$ evolves according to the equation

$$
x_{t+1}=A x_{t}+B u_{t}+w_{t},
$$

where $u_{t} \in \mathbb{R}^{m}$ is the control input and $w_{t} \in \mathbb{R}^{n}$ is a stochastic disturbance with a possibly unbounded support.

We assume that the state of the system is available, and address the problem of designing a state-feedback control policy so as to minimize some finite-horizon cost, while satisfying saturation constraints on the control input and safety constraints on the state. A receding horizon implementation is then possible. In this paper, however, we focus on the finite-horizon problem only.

We adopt the following parametrization for the control input:

$$
u_{i}=\gamma_{i}+\sum_{j=0}^{i-1} \theta_{i, j} \varphi\left(w_{j}\right),
$$

where $\gamma_{i} \in \mathbb{R}^{m}$ and $\theta_{i, j} \in \mathbb{R}^{m \times n}$ are the design parameters, whereas $\varphi: \mathbb{R} \rightarrow \mathbb{R}$ is a given scalar function; with the notation $\varphi\left(w_{j}\right)$, we mean function $\varphi(\cdot)$ applied to each component of the $n$-dimensional vector $w_{j}$. Policy (1) is indeed a state-feedback control policy since the disturbance can be reconstructed from the state measurements according to

$$
w_{i}=x_{i+1}-A x_{i}-B u_{i}
$$

If the scalar function $\varphi(\cdot)$ is the identity map (i.e., $\varphi(\alpha)=$ $\alpha, \forall \alpha \in \mathbb{R}$ ), then we obtain a policy that is affine in the disturbance and that is equivalent to a feedback policy affine

Research supported by the European Commission under the MoVeS project, FP7-ICT-2009-257005

L. Deori, S. Garatti, and M. Prandini are with the Dipartimento di Elettronica, Informazione e Bioingegneria, Politecnico di Milano, Piazza Leonardo da Vinci 32, 20113 Milano, Italy. E-mail: \{deori, sgaratti,prandini\}eelet.polimi.it in the state, [1]. If $\varphi(\cdot)$ is given by the saturation function (or alternatively by a sigmoidal function)

$$
\varphi(\alpha)= \begin{cases}-\bar{\varphi}, & \alpha<-\bar{\varphi} \\ \alpha, & |\alpha| \leq \bar{\varphi} \\ \bar{\varphi}, & \alpha>\bar{\varphi},\end{cases}
$$

then, the resulting policy is a nonlinear function of the state and provides a bounded input even if $w_{j}$ is unbounded, [2]. The advantage of using the control policy (1) is that both the input and the state depend linearly on the parameters $\gamma_{i}$ and $\theta_{i, j}$.

The system performance is expressed in terms of the average quadratic cost

$$
J=\mathbb{E}\left[\sum_{i=1}^{M} x_{i}^{T} Q_{i} x_{i}+\sum_{i=0}^{M-1} u_{i}^{T} R_{i} u_{i}\right],
$$

with $Q_{i}$ and $R_{i}$ symmetric positive semidefinite matrices of appropriate dimensions. We consider constraints of the following form:

$$
\begin{gathered}
\sup _{i=0, \ldots, M-1}\left\|u_{i}\right\|_{\infty} \leq \bar{u}, \\
\sup _{i=1, \ldots, M}\left\|C x_{i}\right\|_{\infty} \leq \bar{y},
\end{gathered}
$$

where the absolute value of all input elements is subject to the same limit $\bar{u}$, and the absolute value of the output variable $y_{i}:=C x_{i}$ is bounded by $\bar{y}$.

When formulating the constraints, one has to account for the uncertainty affecting the system evolution through the noise process $w_{t}$. One can then define either hard or soft constraints: in the first case, constraints must hold for every and each disturbance realization, even for those realizations that are quite unlikely to occur; in the second case, constraints are expressed in probability and must hold on a set of disturbance realizations of probability at least $1-\varepsilon$, with $\varepsilon \in(0,1)$ chosen by the user.

Hard constraints on the control input are typically motivated by the presence of saturation limits of the actuators. However, they may lead to conservative solutions since they do not take into account the statistical properties of the noise. Soft constraints on the input are introduced to reduce this conservatism. In any case, both hard and soft input constraints are always feasible (just take all the design parameters equal to zero to get a feasible point).

As for the state variable, hard constraints are not feasible when the noise distribution has unbounded support, because 
$w_{t}$ enters additively the state equation and this contribution cannot be canceled through any control action. Hence, without assumptions on the boundedness of the noise, one can only head for soft constraints on the state, leading to the following two formulations for the input and state constraints:

- hard \& soft

$$
\left\{\begin{array}{l}
\sup _{i=0, \ldots, M-1}\left\|u_{i}\right\|_{\infty} \leq \bar{u}, \forall\left(w_{0}, w_{1}, \ldots, w_{M-1}\right) \\
\mathbb{P}\left\{\sup _{i=1, \ldots, M}\left\|C x_{i}\right\|_{\infty} \leq \bar{y}\right\} \geq 1-\varepsilon
\end{array}\right.
$$

- soft \& soft

$$
\begin{aligned}
\mathbb{P}\left\{\sup _{i=0, \ldots, M-1}\left\|u_{i}\right\|_{\infty} \leq \bar{u}\right. \\
\left.\quad \wedge \sup _{i=1, \ldots, M}\left\|C x_{i}\right\|_{\infty} \leq \bar{y}\right\} \geq 1-\varepsilon .
\end{aligned}
$$

When formulating the constraints, the value of $\bar{y}$ is quite critical, because of the following two reasons:

- the feasibility of the soft constraint on the state is not always guaranteed since $\bar{y}$ can be not compatible with the disturbance characteristics, the system dynamics, and the saturation limits imposed on the control input;

- even when the soft constraint is feasible, the performance of the obtained solution can be too much adversely affected by the presence of the state constraints if $\bar{y}$ takes a conservative value.

The main thrust of this paper is that, rather than seeing $\bar{y}$ as a fixed value, one should try to modulate it so as to guarantee feasibility, while achieving the appropriate compromise between safety and performance.

More precisely, to address the feasibility issue, $\bar{y}$ is replaced by a decision variable, say $h$, so that it can be automatically set to a value compatible with the system dynamics, input constraints, and noise characteristics.

Then, suitable parametric optimization problems are formulated where both safety and performance are accounted for. The value for the parameter defining the optimization problems determines the compromise between the two objectives of minimizing the control cost $J$ in (3) (performance) and minimizing $h$ (safety). By tuning this parameter, one can explore the different possible trade-offs between safety and performance - while preserving feasibility -, and choose the solution that is more satisfactory in terms of values achieved for $J$ and $h$.

Alternative approaches to tackle the problem when the disturbance has unbounded support have been proposed in, e.g., [3], [4], [5], [6], [7]. In [3], [4], state constraints are replaced by a penalization term accounting for the state constraint violation so as to avoid infeasibility. In [5], [6], [7], a conservative convex relaxation of the chance constraints is adopted, which may result in an infeasible constraint also when the original one was feasible. Derivations are usually confined to the case when the disturbance is a sequence of i.i.d. (independent and identically distributed) random variables and has a Gaussian distribution.

\section{NotATIONAL ISSUES}

Let

$$
\mathbf{x}=\left[\begin{array}{c}
x_{1} \\
x_{2} \\
\vdots \\
x_{M}
\end{array}\right] \quad \mathbf{u}=\left[\begin{array}{c}
u_{0} \\
u_{1} \\
\vdots \\
u_{M-1}
\end{array}\right] \quad \mathbf{w}=\left[\begin{array}{c}
w_{0} \\
w_{1} \\
\vdots \\
w_{M-1}
\end{array}\right]
$$

Then, it is easy to show that

$$
\begin{aligned}
& \mathbf{x}=\mathbf{F} x_{0}+\mathbf{G u}+\mathbf{H} \mathbf{w} \\
& \mathbf{u}=\Gamma+\Theta \varphi(\mathbf{w}),
\end{aligned}
$$

where matrices $\mathbf{F}, \mathbf{G}$ and $\mathbf{H}$ are given by

$$
\begin{gathered}
\mathbf{F}=\left[\begin{array}{c}
A \\
A^{2} \\
\vdots \\
A^{M}
\end{array}\right] \quad \mathbf{G}=\left[\begin{array}{cccc}
B & 0_{n \times m} & \cdots & 0_{n \times m} \\
A B & B & \ddots & \vdots \\
\vdots & \ddots & \ddots & 0_{n \times m} \\
A^{M-1} B & \cdots & A B & B
\end{array}\right] \\
\mathbf{H}=\left[\begin{array}{cccc}
I_{n \times n} & 0_{n \times n} & \cdots & 0_{n \times n} \\
A & I_{n \times n} & \ddots & \vdots \\
\vdots & \ddots & \ddots & 0_{n \times n} \\
A^{M-1} & \cdots & A & I_{n \times n}
\end{array}\right]
\end{gathered}
$$

whereas $\Gamma$ and $\Theta$ are given by

$\Gamma=\left[\begin{array}{c}\gamma_{0} \\ \gamma_{1} \\ \vdots \\ \gamma_{M-1}\end{array}\right] \quad \Theta=\left[\begin{array}{cccc}0_{m \times n} & 0_{m \times n} & \cdots & 0_{m \times n} \\ \theta_{1,0} & 0_{m \times n} & \ddots & \vdots \\ \vdots & \ddots & \ddots & 0_{m \times n} \\ \theta_{M-1,0} & \cdots & \theta_{M-1, M-2} & 0_{m \times n}\end{array}\right]$.

Both $\mathbf{u}$ and $\mathbf{x}$ depend linearly on the parameters $\Gamma$ and $\Theta$.

Let us denote with $\mathbf{Q}=\operatorname{diag}\left(Q_{1}, \ldots, Q_{M}\right)$ and $\mathbf{R}=$ $\operatorname{diag}\left(R_{0}, \ldots, R_{M-1}\right)$ the block diagonal matrices built based on $Q_{i}$ and $R_{i}$ in (3). If we set $m_{\mathbf{w}}=\mathbb{E}[\mathbf{w}], m_{\varphi}=\mathbb{E}[\varphi(\mathbf{w})]$ and

$$
\mathbf{V}=\left[\begin{array}{cc}
V_{\varphi \varphi} & V_{\varphi \mathbf{w}} \\
V_{\varphi \mathbf{w}}^{T} & V_{\mathbf{w w}}
\end{array}\right]
$$

where $V_{\mathbf{w w}}$ and $V_{\varphi \varphi}$ are the covariance matrices of $\mathbf{w}$ and $\varphi(\mathbf{w})$ and $V_{\varphi \mathbf{w}}$ is the cross-covariance matrix of $\varphi(\mathbf{w})$ and $\mathbf{w}$, then, the control cost (3) can be expressed as the following convex function of $(\Gamma, \Theta)$ :

$$
\begin{aligned}
& J(\Gamma, \Theta)=\mathbb{E}\left[\mathbf{x}^{T} \mathbf{Q} \mathbf{x}+\mathbf{u}^{T} \mathbf{R u}\right] \\
& =\left(\mathbf{F} x_{0}+\mathbf{G} \Gamma+\mathbf{G} \Theta m_{\varphi}+\mathbf{H} m_{\mathbf{w}}\right)^{T} \mathbf{Q}\left(\mathbf{F} x_{0}+\right. \\
& \left.+\mathbf{G} \Gamma+\mathbf{G} \Theta m_{\varphi}+\mathbf{H} m_{\mathbf{w}}\right)+
\end{aligned}
$$

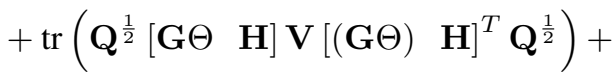

$$
\begin{aligned}
& +\left(\Gamma+\Theta m_{\varphi}\right)^{T} \mathbf{R}\left(\Gamma+\Theta m_{\varphi}\right)+\operatorname{tr}\left(\mathbf{R}^{\frac{1}{2}} \Theta V_{\varphi \varphi} \Theta^{T} \mathbf{R}^{\frac{1}{2}}\right)
\end{aligned}
$$

As for the constraints (4), if we set

$$
\mathbf{C}=\operatorname{diag}(C, \ldots, C)
$$

then, they can be expressed in compact form as follows:

$$
\|\mathbf{u}\|_{\infty} \leq \bar{u} \quad\|\mathbf{C x}\|_{\infty} \leq h,
$$


where $\bar{y}$ has been replaced with the optimization variable $h$. Thanks to the linear dependence of $\mathbf{u}$ and $\mathbf{x}$ on $\Gamma$ and $\Theta$, these constraints turn out to be convex with respect to the optimization variables.

\section{APPROACH BASED ON CONTROL COST PENALIZATION}

In this first method, a penalization term is added to the average quadratic cost $J$ in (3) in order to account for the bound $h$ on the state:

$$
J^{\prime}=J+\mu h .
$$

The coefficient $\mu \geq 0$ determines the trade-off between the two objectives $J$ (performance) and $h$ (safety). Depending on the kind of constraint adopted for the control input, two chance-constrained problems can be formulated:

$$
\begin{gathered}
\min _{\Gamma, \Theta, h} J(\Gamma, \Theta)+\mu h \text { subject to: } \\
\left\{\begin{array}{l}
\|\mathbf{u}\|_{\infty} \leq \bar{u}, \forall \mathbf{w} \\
\mathbb{P}\left\{\|\mathbf{C x}\|_{\infty} \leq h\right\} \geq 1-\varepsilon
\end{array}\right. \\
\min _{\Gamma, \Theta, h} J(\Gamma, \Theta)+\mu h \text { subject to: } \\
\mathbb{P}\left\{\|\mathbf{u}\|_{\infty} \leq \bar{u} \wedge\|\mathbf{C x}\|_{\infty} \leq h\right\} \geq 1-\varepsilon
\end{gathered}
$$

\section{TWO-STEP APPROACH BASED ON A PRE-DEFINED} ADMISSIBLE DETERIORATION OF THE CONTROL COST

In this second approach, feasibility is still recovered by considering the bound on the state as an optimization variable. However, the two objectives of minimizing the control cost as well as the bound on the state are handled differently. More precisely, two optimization problems are solved in cascade: in the first one, the control cost is minimized subject only to the control input constraints, whereas in the second one, $h$ is minimized subject to the constraints on both state and control input and a further constraint on the maximum admissible degradation of the control cost with respect to the value $J^{\star}$ computed in the first problem: $J \leq J^{\star}+\alpha$, with $\alpha \geq 0$. Again, the coefficient $\alpha$ determines the trade-off between the objective of minimizing $J$ (performance) and that of minimizing $h$ (safety).

In the case when hard constraints are imposed on the control input, the first optimization problem is given by

$$
\begin{aligned}
& \min _{\Gamma, \Theta} J(\Gamma, \Theta) \text { subject to: } \\
& \|\mathbf{u}\|_{\infty} \leq \bar{u} \quad \forall \mathbf{w},
\end{aligned}
$$

while, letting $J^{\star}$ be the optimal cost obtained by solving (8a), the second optimization problem is:

$$
\begin{aligned}
& \min _{\Gamma, \Theta, h} h \quad \text { subject to: } \\
& \left\{\begin{array}{l}
\|\mathbf{u}\|_{\infty} \leq \bar{u} \quad \forall \mathbf{w} \\
\mathbb{P}\left\{\|\mathbf{C x}\|_{\infty} \leq h\right\} \geq 1-\varepsilon . \\
J(\Gamma, \Theta) \leq J^{\star}+\alpha
\end{array}\right.
\end{aligned}
$$

If the control input is subject to a probabilistic constraint as well, then, the first optimization problem writes

$$
\begin{aligned}
& \min _{\Gamma, \Theta} J(\Gamma, \Theta) \text { subject to: } \\
& \mathbb{P}\left\{\|\mathbf{u}\|_{\infty} \leq \bar{u}\right\} \geq 1-\varepsilon,
\end{aligned}
$$

while, letting $J^{\star}$ denote the optimal cost obtained by solving (9a), the second optimization problem is:

$$
\begin{aligned}
& \min _{\Gamma, \Theta, h} h \quad \text { subject to: } \\
& \left\{\begin{array}{l}
\mathbb{P}\left\{\|\mathbf{u}\|_{\infty} \leq \bar{u} \wedge\|\mathbf{C x}\|_{\infty} \leq h\right\} \geq 1-\varepsilon \\
J(\Gamma, \Theta) \leq J^{\star}+\alpha
\end{array} .\right.
\end{aligned}
$$

\section{APPROXIMATE SOLUTION}

The problems posed in Sections III and IV are known to be hard to solve.

Although the robust constraint $\|\mathbf{u}\|_{\infty} \leq \bar{u}, \forall \mathbf{w}$, is convex given the chosen parametrization of $\mathbf{u}$, it requires to take into account the whole infinite amount of possible realizations of the noise process $\mathbf{w}$, raising the problem complexity an order of magnitude over standard convex optimization. The probabilistic constraints $\mathbb{P}\left\{\|\mathbf{C x}\|_{\infty} \leq h\right\} \geq 1-\varepsilon$ and $\mathbb{P}\left\{\|\mathbf{u}\|_{\infty} \leq \bar{u} \wedge\|\mathbf{C x}\|_{\infty} \leq h\right\} \geq 1-\varepsilon$, instead, are even non-convex in general, though $\|\mathbf{u}\|_{\infty} \leq \bar{u}$ and $\|\mathbf{C x}\|_{\infty} \leq h$ are convex for any fixed realization of $\mathbf{w}$.

In this section, suitable relaxations of problems (6)-(9), which are amenable to be solved by means of standard convex optimization techniques, are introduced and discussed.

\section{A. Algorithms}

Clearly, the issue is how to tackle the robust and the probabilistic constraints appearing in the various problems. Being the support of $\mathbf{w}$ unbounded, we assume that in presence of the robust constraint $\|\mathbf{u}\|_{\infty} \leq \bar{u}, \forall \mathbf{w}$, function $\varphi(\cdot)$ is chosen as a saturation function as in (2) because otherwise, if e.g. $\varphi(\cdot)$ is the identity map, the robust constraint would always lead to solutions with $\Theta=0$, i.e., to a control policy without the feedback term. Following [2], [8], the robust constraint is then replaced by the following finite set of constraints:

$$
\left|\Gamma_{i}\right|+\left\|\Theta_{i}\right\|_{1} \bar{\varphi} \leq \bar{u}, i=1, \ldots, m M,
$$

where $\Gamma_{i}$ denotes the $i$-th element of vector $\Gamma$ and $\Theta_{i}$ the $i$ th row of $\Theta$. The idea behind (10) is that $\mathbf{u}$ cannot be worse than when the components of $\varphi(\mathbf{w})$ have all absolute value equal to $\bar{\varphi}$ and signs such that the elements of each row $\Theta_{i} \varphi(\mathbf{w})+\Gamma_{i}$ positively sum up. Plainly, any feasible point for the newly introduced set of constraints is also feasible for the original robust constraint. The number of constraints in (10) is finite and usually small and (10) can be dealt with by means of standard solvers.

As for the probabilistic constraints, we resort to the scenario approach, a recently developed randomized method to approximately solved chance-constrained problems, [9], [10], [11], [12], [13]. 
The idea behind the scenario approach is very simple. A bunch of $N$ realizations of the disturbance $\mathbf{w}$, say $\mathbf{w}^{(1)}, \mathbf{w}^{(2)}, \ldots, \mathbf{w}^{(N)}$, is generated according to the underlying probability distribution of $\mathbf{w}$. Then, the probabilistic constraints are replaced with a finite number $N$ of constraints of the type $\|\mathbf{C x}\|_{\infty} \leq h$ and/or $\|\mathbf{u}\|_{\infty} \leq \bar{u}$, those obtained in correspondence of the generated instances of the disturbance. More precisely, writing explicitly the dependence of $\mathbf{x}$ and $\mathbf{u}$ on $\mathbf{w}$, the constraint

$$
\mathbb{P}\left\{\|\mathbf{C x}(\mathbf{w})\|_{\infty} \leq h\right\} \geq 1-\varepsilon
$$

is replaced by

$$
\left\|\mathbf{C x}\left(\mathbf{w}^{(i)}\right)\right\|_{\infty} \leq h, \quad i=1, \ldots, N
$$

while

$$
\mathbb{P}\left\{\|\mathbf{u}\|_{\infty} \leq \bar{u} \wedge\|\mathbf{C x}\|_{\infty} \leq h\right\} \geq 1-\varepsilon
$$

is replaced by

$$
\left\{\begin{array}{l}
\left\|\mathbf{u}\left(\mathbf{w}^{(i)}\right)\right\|_{\infty} \leq \bar{u} \\
\left\|\mathbf{C x}\left(\mathbf{w}^{(i)}\right)\right\|_{\infty} \leq h
\end{array} \quad i=1, \ldots, N\right.
$$

Summarizing, depending on the chosen type of constraint (robust or in probability) for the input, and on the chosen method to take into account the presence of the optimization variable $h$, the possible reformulations are the following four.

- Cost penalization and hard constraint on input:

$$
\begin{aligned}
& \min _{\Gamma, \Theta, h} J(\Gamma, \Theta)+\mu h \quad \text { subject to: } \\
& \left\{\begin{array}{l}
\left|\Gamma_{i}\right|+\left\|\Theta_{i}\right\|_{1} \bar{\varphi} \leq \bar{u}, \quad i=1, \ldots, m M \\
\left\|\mathbf{C x}\left(\mathbf{w}^{(i)}\right)\right\|_{\infty} \leq h, \quad i=1, \ldots, N
\end{array}\right.
\end{aligned}
$$

- Cost penalization and soft constraint on input:

$$
\begin{aligned}
& \min _{\Gamma, \Theta, h} J(\Gamma, \Theta)+\mu h \quad \text { subject to: } \\
& \left\{\begin{array}{l}
\left\|\mathbf{u}\left(\mathbf{w}^{(i)}\right)\right\|_{\infty} \leq \bar{u} \\
\left\|\mathbf{C x}\left(\mathbf{w}^{(i)}\right)\right\|_{\infty} \leq h
\end{array}\right.
\end{aligned}
$$

- Two-step approach and hard constraint on input:

$$
\begin{aligned}
& \min _{\Gamma, \Theta} J(\Gamma, \Theta) \quad \text { subject to: } \\
& \left|\Gamma_{i}\right|+\left\|\Theta_{i}\right\|_{1} \bar{\varphi} \leq \bar{u}, \quad i=1, \ldots, m M
\end{aligned}
$$

Let $J^{\star}$ be the optimal cost value of (13a).

$$
\begin{aligned}
& \min _{\Gamma, \Theta, h} h \quad \text { subject to: } \\
& \left\{\begin{array}{l}
\left|\Gamma_{i}\right|+\left\|\Theta_{i}\right\|_{1} \bar{\varphi} \leq \bar{u}, \quad i=1, \ldots, m M, \\
\left\|\mathbf{C x}\left(\mathbf{w}^{(i)}\right)\right\|_{\infty} \leq h, \quad i=1, \ldots, N \\
J(\Gamma, \Theta) \leq J^{\star}+\alpha
\end{array}\right.
\end{aligned}
$$

- Two-step approach and soft constraint on input:

$$
\begin{aligned}
& \min _{\Gamma, \Theta} J(\Gamma, \Theta) \quad \text { subject to: } \\
& \left\|\mathbf{u}\left(\mathbf{w}^{(i)}\right)\right\|_{\infty} \leq \bar{u} \quad i=1, \ldots, N
\end{aligned}
$$

Let $J^{\star}$ be the optimal cost value of (14a).

$$
\begin{aligned}
& \min _{\Gamma, \Theta, h} h \quad \text { subject to: } \\
& \left\{\begin{array}{l}
\left\{\begin{array}{l}
\left\|\mathbf{u}\left(\mathbf{w}^{(i)}\right)\right\|_{\infty} \leq \bar{u} \\
\left\|\mathbf{C} \mathbf{x}\left(\mathbf{w}^{(i)}\right)\right\|_{\infty} \leq h \\
J(\Gamma, \Theta) \leq J^{\star}+\alpha
\end{array} \quad i=1, \ldots, N\right.
\end{array}\right.
\end{aligned}
$$

As for this latter problem, note that the same realizations $\mathbf{w}^{(1)}, \mathbf{w}^{(2)}, \ldots \mathbf{w}^{(N)}$ must be used both in (14a) and in (14b), because, otherwise, there are no guarantees that the program in (14b) (which is based on the solution of (14a)) is feasible. All problems (11)-(14) consist in solving convex programs with a finite number of constraints that require no machinery other than standard convex optimization solvers like those used by CVX, [14], and YALMIP, [15]. Moreover, despite the apparent naivety of the scenario approach, the obtained solution comes with precise guarantees about its feasibility with respect the original probabilistic constraint as long as $N$ is suitably chosen. This is discussed in the next section.

\section{B. Feasibility of the obtained approximate solutions}

The problems (11)-(14) are obtained as relaxations of the original problems (6)-(9). The sub-optimality of the obtained solutions is the price we must pay to enhance computational tractability. However, a main issue is whether the solutions to problems (11), (12), (13), and (14) are feasible for the original constraints on $\mathbf{u}$ and $\mathbf{x}$ in problems (6), (7), (8), and (9), respectively.

As already discussed, the relaxation introduced for the robust constraint is such that feasibility with respect to the original hard constraint is preserved. The same issue arises for the relaxation of the constraint in probability introduced by the scenario approach, but is much more involved to address. The following theorem ${ }^{1}$ provides a fundamental result in this respect.

Theorem 1: Let $f(\xi): \mathbb{R}^{d} \rightarrow \mathbb{R}$ be a convex function and $g(\xi, \delta): \mathbb{R}^{d} \times \Delta \rightarrow \mathbb{R}$ be a parametric family of convex functions (i.e. $g(\xi, \delta)$ is convex in $\xi$ for any fixed value of $\delta \in \Delta)$. Moreover, let $\Xi$ be any given convex subset of $\mathbb{R}^{d}$. For any $\varepsilon \in(0,1)$ and $\beta \in(0,1)$, if

$$
N \geq \frac{d+1+\ln (1 / \beta)+\sqrt{2(d+1) \ln (1 / \beta)}}{\varepsilon},
$$

then, the solution $\xi^{*}$ of problem

$$
\begin{aligned}
& \min _{\xi \in \Xi \subseteq \mathbb{R}^{d}} f(\xi) \quad \text { subject to: } \\
& g\left(\xi, \delta^{(i)}\right) \leq 0, \quad i=1, \ldots, N,
\end{aligned}
$$

where $\delta^{(1)}, \delta^{(2)}, \ldots, \delta^{(N)}$ are samples independently extracted according to a given probability $\mathbb{P}_{\delta}$ over $\Delta$, is feasible for the constraint in probability

$$
\mathbb{P}_{\delta}\{g(\xi, \delta) \leq 0\} \geq 1-\varepsilon,
$$

with high confidence $1-\beta$.

\footnotetext{
${ }^{1}$ Theorem 1 is proven in [11], though an implicit expression for $N$ is given. The explicit expression used here is due to [16].
} 
Note that the feasibility of $\xi^{*}$ for the probabilistic constraint can be guaranteed with high confidence $1-\beta$ only. This is intrinsically so because $\xi^{*}$ is random as it depends on the extracted $\delta^{(1)}, \delta^{(2)}, \ldots, \delta^{(N)}$. However, $N$ depends on $\beta$ logarithmically so that small values of $\beta$ like $\beta=10^{-5}$ or $\beta=10^{-7}$ can be forced in without affecting $N$ too much. With such values for $\beta$, the result in Theorem 1 reads as " $\xi$ " is feasible for the constraint in probability beyond any reasonable doubt".

A direct application of Theorem 1 to (11), (12), (13b) ${ }^{2}$ shows that, if $N$ satisfies (15), then the solutions of Problems (11), (12), (13) are feasible with high confidence $1-\beta$ for the constraints on $\mathbf{u}$ and $\mathbf{x}$ in (6), (7), (8), respectively.

As for Problem (14), Theorem 1 does not apply in this case. As a matter of fact, $J^{\star}$ in (14b) should be more properly written as $J^{\star}\left(\mathbf{w}^{(1)}, \mathbf{w}^{(2)}, \ldots, \mathbf{w}^{(N)}\right)$, being obtained as the optimal value of (14a), a program where constraints depend on $\mathbf{w}^{(1)}, \mathbf{w}^{(2)}, \ldots, \mathbf{w}^{(N)}$. This means that $\Xi=$ $\Xi\left(\mathbf{w}^{(1)}, \mathbf{w}^{(2)}, \ldots, \mathbf{w}^{(N)}\right)$, a setup which is not covered by Theorem 1.

Although we experimentally verified that, for $N$ large enough, the solution of Problem (14) is usually feasible for the constraint in probability

$$
\mathbb{P}\left\{\|\mathbf{u}\|_{\infty} \leq \bar{u} \wedge\|\mathbf{C x}\|_{\infty} \leq h\right\} \geq 1-\varepsilon
$$

we were not able to prove that feasibility holds with high confidence $1-\beta$ for $N$ satisfying (15). This latter property for the solution to Problem (14) remains a conjecture.

\section{Choice of $\mu \& \alpha$ and trade-off between $J \& h$}

As in Problems (6)-(9), the parameters $\mu$ and $\alpha$ in Problems (11)-(14) play the role of tuning parameters through which the user can modulate the trade-off between the objective of minimizing the control cost $J$ and that of having a small $h$ to strengthen the safety of the system operation.

Heuristically, one can proceed by solving the chosen program among (11), (12), (13), and (14) for a grid of values of $\mu$ or $\alpha$, say $\mu_{1}, \mu_{2}, \ldots, \mu_{k}$ or $\alpha_{1}, \alpha_{2}, \ldots, \alpha_{k}$, each time using the same realizations $\mathbf{w}^{(1)}, \mathbf{w}^{(2)}, \ldots, \mathbf{w}^{(N)}$ of the noise. This way, various solutions are obtained, each returning a different trade-off between $J$ and $h$. By inspecting the achieved values, the best solution according to the problem at hand can be eventually selected.

Under the assumption that the matrices $\mathbf{R}$ and $V_{\varphi \varphi}=$ $\mathbb{E}\left[(\varphi(\mathbf{w})-\mathbb{E}[\varphi(\mathbf{w})])\left(\varphi(\mathbf{w})-\mathbb{E}[\varphi(\mathbf{w}))^{T}\right]\right.$ are positive definite it can be shown, [17], that $\mu$ and $\alpha$ are sensible tuning parameters since by progressively increasing them from 0 to

$$
\begin{gathered}
{ }^{2} \text { Let } \xi=(\Gamma, \Theta, h), \delta=\mathbf{w}, \\
f(\xi)= \begin{cases}J(\Gamma, \Theta)+\mu h & \text { for (11),(12) } \\
h & \text { for (13b) },\end{cases} \\
g\left(\xi, \delta^{(i)}\right)= \begin{cases}\left\|\mathbf{C x}\left(\mathbf{w}^{(i)}\right)\right\|_{\infty}-h & \text { for (11),(13b) } \\
\max \left(\begin{array}{l}
\left\|\mathbf{u}\left(\mathbf{w}^{(i)}\right)\right\|_{\infty}-\bar{u}, \\
\left\|\mathbf{C x}\left(\mathbf{w}^{(i)}\right)\right\|_{\infty}-h
\end{array}\right) & \text { for (12) }\end{cases}
\end{gathered}
$$

and $\Xi$ be defined by the remaining constraints, which do not depend on $\mathbf{w}^{(i)}$. $\infty$, all possible trade-offs between the two extremes given by the solutions to problems

$$
\begin{aligned}
& \min _{\Gamma, \Theta, h} J(\Gamma, \Theta) \quad \text { subject to: } \\
& F\left(\Gamma, \Theta, h, \mathbf{w}^{(1)}, \ldots, \mathbf{w}^{(N)}\right) \leq 0
\end{aligned}
$$

and

$$
\begin{aligned}
& \min _{\Gamma, \Theta, h} h \quad \text { subject to: } \\
& F\left(\Gamma, \Theta, h, \mathbf{w}^{(1)}, \ldots, \mathbf{w}^{(N)}\right) \leq 0
\end{aligned}
$$

can be achieved. This also reveals a substantial equivalence between the two approaches.

\section{NUMERICAL EXAMPLE}

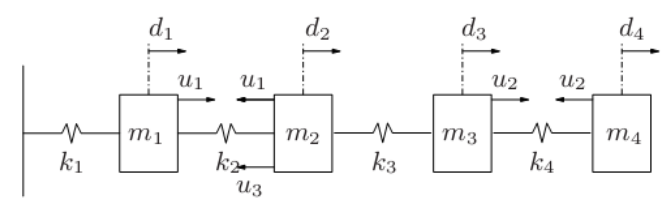

Fig. 1. Scheme of the mechanical system.

We consider the mechanical system represented in Figure 1 which is composed of four masses and four springs, [7]. The state of the system is given by the mass displacements from the equilibrium position achieved when all inputs are null and by their derivatives: $x=\left[d_{1}, d_{2}, d_{3}, d_{4}, \dot{d}_{1}, \dot{d}_{2}, \dot{d}_{3}, \dot{d}_{4}\right]^{T}$. The control input is $u=\left[u_{1}, u_{2}, u_{3}\right]^{T}$ where $u_{1}, u_{2}$ and $u_{3}$ are forces acting on the masses as in Figure 1.

We set all masses and stiffness constants equal to 1, i.e., $m_{1}=m_{2}=m_{3}=m_{4}=1$ and $k_{1}=k_{2}=k_{3}=k_{4}=1$, and consider a discrete time model of the system,

$$
x_{t+1}=A x_{t}+B u_{t}+w_{t},
$$

obtained by time discretization of a standard mechanical model under the assumption that the control action is piecewise constant over the intervals $[t, t+1)$ and neglecting friction forces. We suppose that the state of the system is affected by a white Gaussian noise $w$ with zero mean and variance $\sigma^{2} I_{8 \times 8}, \sigma=1$.

Our goal is to design a state feedback control policy that is able to counteract the disturbance $w$, maintaining the system mode close to the equilibrium and keeping the springs within their linear operating domain. This constraint is imposed by requiring the spring deformations to be small.

To the purpose of regulating the system around the equilibrium, we consider the average control cost (3) with a prediction horizon of length $M=5$ and constant weight matrices

$$
Q=\left[\begin{array}{ll}
I_{4 \times 4} & 0_{4 \times 4} \\
0_{4 \times 4} & 0_{4 \times 4}
\end{array}\right] \quad R=10^{-6} I_{3 \times 3} .
$$

Let $C$ be such that

$$
C x_{i}=\left[\begin{array}{c}
d_{1, i} \\
d_{2, i}-d_{1, i} \\
d_{3, i}-d_{2, i} \\
d_{4, i}-d_{3, i}
\end{array}\right],
$$


i.e. $C x_{i}$ provides the springs deformation at time $i$. Then, the state constraints, introduced to limit the springs deformation, can be expressed as $\|\mathbf{C x}\|_{\infty} \leq h$, where $\mathbf{C}$ is defined as in (5). Eventually, we suppose that the control input is subject to the saturation limit $\|\mathbf{u}\|_{\infty} \leq \bar{u}$, where $\bar{u}=5$.

The control policy is parameterized according to (1) where $\varphi(\cdot)$ is the saturation function in (2) with $\bar{\varphi}$ set equal to $2 \sigma$. The initial state is zero, i.e., the system starts at the equilibrium point. We focus on the case when constraints on both the control input and the state are expressed in probability with an admissible violation $\varepsilon=0.1$. The resulting chanceconstrained optimization problems are solved through the scenario approach by setting $\beta=10^{-5}$. Correspondingly, the number of disturbance realizations to extract is $N=3455$. Note that, since we are imposing a probabilistic bound on the control input, there may be disturbance realizations such that the bound on $\mathbf{u}$ is violated. In that case, the components of $\mathbf{u}$ whose absolute value exceeds $\bar{u}$ are saturated to $\pm \bar{u}$ (clipping of the control input) and performance in terms of the average control cost (3) is estimated via Monte Carlo simulation.

Both the approaches of Section III (control cost penalization) and of Section IV (two-step approach) were adopted by solving scenario programs by means of YALMIP over SeDuMi, [15]. However according to their equivalence discussed in Section V-C the two approaches led to the same results and, hence, Table I reports the results achieved by the control cost penalization approach only.

From Table I, it is apparent that parameter $\mu$ affects the trade-off between the two objectives, i.e., control cost and state constraints: for small $\mu$ values, the state constraints are ineffective in practice, whereas for large $\mu$ 's, $h$ decreases at the price of a significant increase of $J$.

The performance $\hat{\bar{J}}$ of the clipped scenario solution as well as the estimate $\hat{\varepsilon}$ of the actual probability of constraint violation are estimated through 5000 runs of the controlled system. It is worth noticing that the estimate $\hat{\varepsilon}$ is always smaller than $\varepsilon=0.1$, as it is guaranteed by the scenario theory with confidence $1-10^{-5}$. As a result, the sample estimate $\hat{\bar{J}}$ is quite close to the value $J$ obtained when solving the scenario optimization problem.

TABLE I

APPROACH WITH PENALIZATION OF THE CONTROL COST.

\begin{tabular}{|r|r|r|r|r|}
\hline$\mu$ & $J$ & $h$ & $\hat{\bar{J}}$ & $\hat{\varepsilon}$ \\
\hline 0 & 37.29 & 9.05 & 37.49 & 0.0298 \\
0.1 & 37.31 & 8.57 & 37.51 & 0.0324 \\
1 & 38.00 & 7.15 & 38.15 & 0.0322 \\
10 & 43.37 & 5.78 & 43.37 & 0.0520 \\
100 & 48.94 & 5.41 & 48.66 & 0.0942 \\
\hline
\end{tabular}

If constraints on both state and control input were ignored, one would get the optimal LQG control policy that is linear in the state. In the case of clipped LQG control, the estimated control cost $\hat{\bar{J}}$ is 45.79 , with a significant degradation with respect to the optimal LQG cost in absence of input constraints which is 30.76. As for the state constraints the estimate of the minimum $h$ satisfying $\mathbb{P}\left\{\|\mathbf{C x}\|_{\infty} \leq h\right\} \geq 1-\varepsilon$ for the clipped LQG is 9.35. Except for the last row in Table I the control cost penalization approach outperforms clipped LQG in terms of value of the control cost, while at the same time improving over the bound $h$ on the state, since state constraints are explicitly accounted for in the control design.

\section{REFERENCES}

[1] P. Goulart, E. Kerrigan, and J. Maciejowski, "Optimization over state feedback policies for robust control with constraints," Automatica, vol. 42, no. 4, pp. 523-533, April 2006.

[2] P. Hokayem, D. Chatterjee, and J. Lygeros, "On stochastic model predictive control with bounded control inputs," in IEEE Conference on Decision and Control, Shanghai, China, December 16-18, 2009.

[3] I. Batina, A. Stoorvogel, and S. Weiland, "Optimal control of linear, stochastic systems with state and input constraints," in Proc. of the 41st IEEE Conference on Decision and Control, Dec. 2002.

[4] J. Primbs, "A soft constraint approach to stochastic receding horizon control," in Proc. of the 46th IEEE Conference on Decision and Control, Dec. 2007.

[5] D. V. Hessem and O. Bosgra, "Stochastic closed-loop model predictive control of continuous nonlinear chemical processes," Journal of Process Control, vol. 16, no. 3, pp. 225 - 241, 2006.

[6] M. Ono and B. C. Williams, "Iterative risk allocation: A new approach to robust model predictive control with a joint chance constraint," in Proc. of the 47th IEEE Conference on Decision and Control, Dec. 2008.

[7] E. Cinquemani, M. Agarwal, D. Chatterjee, and J. Lygeros, "Convexity and convex approximations of discrete-time stochastic control problems with constraints," Automatica, vol. 47, no. 9, pp. 2082-2087, 2011.

[8] P. Hokayem, E. Cinquemani, D. Chatterjee, F. Ramponi, and J. Lygeros, "Stochastic receding horizon control with output feedback and bounded controls," Automatica, vol. 48, no. 1, pp. 77-88, January 2012.

[9] G. Calafiore and M. Campi, "Uncertain convex programs: randomized solutions and confidence levels," Mathematical Programming, vol. 102, no. 1, pp. 25-46, 2005.

[10] - "The scenario approach to robust control design," IEEE Transactions on Automatic Control, vol. 51, no. 5, pp. 742-753, 2006.

[11] M. Campi and S. Garatti, "The exact feasibility of randomized solutions of uncertain convex programs," SIAM Journal on Optimization, vol. 19 , no. 3 , pp. 1211-1230, 2008 .

[12] M. Campi, S. Garatti, and M. Prandini, "The scenario approach for systems and control design," Annual Reviews in Control, vol. 33, no. 2, pp. 149-157, 2009.

[13] M. Campi and S. Garatti, "A sampling-and-discarding approach to chance-constrained optimization: Feasibility and optimality," Journal of Optimization Theory and Applications, vol. 148, no. 2, pp. 257-280, 2011.

[14] M. Grant and S. Boyd, "CVX: Matlab software for disciplined convex programming, version 1.21," http: / / cvxr.com/cvx, Feb 2011.

[15] J. Löfberg, "Yalmip: a toolbox for modeling and optimization in MATLAB," in Proc. of the CACSD Conference, Taipei, Taiwan, 2004. [Online]. Available: http://control.ee.ethz.ch/ joloef/yalmip.php

[16] T. Alamo, R. Tempo, and A. Luque, "On the sample complexity of randomized approaches to the analysis and design under uncertainty," in Proc. of the 2010 Amer. Contr. Conf., Baltimore, MD, USA, Jun. 2010, pp. 4671-4676.

[17] L. Deori, "Stochastic optimal control in presence of constraints," Master's thesis, Politecnico di Milano, Milano, Italy, September 2012, in Italian. 